\title{
ARTICLE
}

Multiple Myeloma Gammopathies

\section{Copy number evolution and its relationship with patient outcome-an analysis of 178 matched presentation-relapse tumor pairs from the Myeloma XI trial}

\author{
James Croft ${ }^{1} \cdot$ Sidra Ellis $^{1} \cdot$ Amy L. Sherborne ${ }^{1} \cdot$ Kim Sharp $^{1} \cdot$ Amy Price $^{1} \cdot$ Matthew W. Jenner $^{2}$.

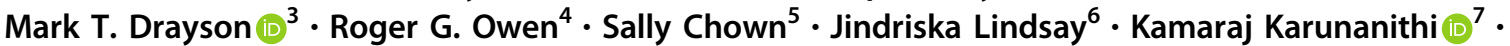 \\ Hannah Hunter $^{8}$ - Walter M. Gregory $\mathbb{D}^{9} \cdot$ Faith E. Davies $^{10} \cdot$ Gareth J. Morgan $^{10}$ • Gordon Cook $\mathbb{B}^{11}$.

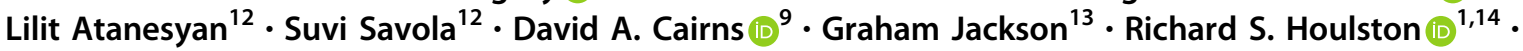 \\ Martin F. Kaiser (iD ${ }^{1,15}$
}

Received: 30 June 2020 / Revised: 2 October 2020 / Accepted: 14 November 2020 / Published online: 1 December 2020

(c) The Author(s) 2020. This article is published with open access

\begin{abstract}
Structural chromosomal changes including copy number aberrations (CNAs) are a major feature of multiple myeloma (MM), however their evolution in context of modern biological therapy is not well characterized. To investigate acquisition of CNAs and their prognostic relevance in context of first-line therapy, we profiled tumor diagnosis-relapse pairs from 178 NCRI Myeloma XI (ISRCTN49407852) trial patients using digital multiplex ligation-dependent probe amplification. CNA profiles acquired at relapse differed substantially between MM subtypes: hyperdiploid (HRD) tumors evolved predominantly in branching pattern vs. linear pattern in $\mathrm{t}(4 ; 14)$ vs. stable pattern in $\mathrm{t}(11 ; 14)$. CNA acquisition also differed between subtypes based on $C C N D$ expression, with a marked enrichment of acquired $\operatorname{del}(17 \mathrm{p})$ in $C C N D 2$ over $C C N D 1$ tumors. Acquired CNAs were not influenced by high-dose melphalan or lenalidomide maintenance randomization. A branching evolution pattern was significantly associated with inferior overall survival (OS; hazard ratio (HR) 2.61, $P=0.0048$ ). As an individual lesion, acquisition of gain(1q) at relapse was associated with shorter OS, independent of other risk markers or time of relapse $(\mathrm{HR}=2.00 ; P=0.021)$. There is an increasing need for rational therapy sequencing in MM. Our data supports the value of repeat molecular profiling to characterize disease evolution and inform management of MM relapse.
\end{abstract}

\section{Introduction}

Supplementary information The online version of this article (https:// doi.org/10.1038/s41375-020-01096-y) contains supplementary material, which is available to authorized users.

Martin F. Kaiser

Martin.Kaiser@icr.ac.uk

1 Division of Molecular Pathology, The Institute of Cancer Research, London, UK

2 Department of Haematology, University Hospital Southampton NHS Foundation Trust, Southampton, UK

3 Institute of Immunology and Immunotherapy, University of Birmingham, Birmingham, UK

4 Haematological Malignancy Diagnostic Service, St. James's University Hospital, Leeds, UK

5 Gloucestershire Hospitals NHS Foundation Trust, Gloucester, UK

6 Kent and Canterbury Hospital, Canterbury, UK

7 University Hospital of North Midlands, Stoke-on-Trent, UK
Multiple myeloma (MM) is caused by the malignant clonal expansion of plasma cells in the bone marrow [1].

8 Derriford Hospital, Plymouth, UK

9 Clinical Trials Research Unit, Leeds Institute of Clinical Trials Research, Leeds, UK

10 Perlmutter Cancer Center, NYU Langone Health, New York, NY, USA

11 Leeds Institute of Cancer and Pathology, University of Leeds, Leeds, UK

12 MRC-Holland, Amsterdam, The Netherlands

13 Department of Haematology, University of Newcastle, Newcastle Upon Tyne, UK

14 Division of Genetics and Epidemiology, The Institute of Cancer Research, London, UK

15 Department of Hematology, The Royal Marsden Hospital, London, UK 
Approximately $40 \%$ of $\mathrm{MM}$ tumors harbor chromosome $\mathrm{t}(4 ; 14), \mathrm{t}(11 ; 14)$, or $\mathrm{t}(14 ; 16) / \mathrm{t}(14 ; 20)$ translocations, which result in overexpression of oncogenes (including WHSC1/ $M M S E T / N S D 2, F G F R 3, C C N D 1, M A F$, and MAFB) through juxtaposition to the immunoglobulin heavy-chain locus [2, 3]. Other MM tumors exhibit hyperdiploidy (HRD), which is considered an alternative initiating event. Increased expression of CCNDI in t $(11 ; 14)$ and HRD and increased $C C N D 2$ expression in $\mathrm{t}(4 ; 14), \mathrm{t}(14 ; 16) / \mathrm{t}(14 ; 20)$, and HRD are unifying downstream consequences of initiating lesions [4-10].

Recent next-generation sequencing projects have provided insight into the sub-clonal complexity of tumor progression in MM [11-14]. However, few recurrent single nucleotide changes have been reported to be associated with disease relapse, notably sub-clonal mutations of cereblon in immunomodulatory drug (IMiD) treated patients. Knowledge about their prognostic impact remains limited [15]. In contrast, several large-scale chromosomal abnormalities, including gain(1q), del(1p), and $\operatorname{del}(17 \mathrm{p})$ have been shown to be acquired during tumor progression, but their association with tumor subtype, treatment and outcome is not well understood $[11,16]$. Recent studies of the relationship between copy number aberrations (CNAs) and progression of MM have largely been based on shortread sequencing or FISH. Importantly, few studies have been performed on patients from a randomized clinical trial and the impact of therapy on acquisition of CNAs remains unclear [15, 16]. To gain insight into the relationship between CNAs and progression of MM we performed virtual karyotyping of all chromosomes and targeted hotspot profiling using digital multiplexed ligation-dependent probe amplification (digitalMLPA) in matched diagnosis-relapse tumor pairs in 178 UK NCRI Myeloma $\mathrm{XI}$ trial patients [17]. We show that acquisition of CNAs is strongly correlated with MM subgroups. Additionally, a number of acquired changes have independent predictive value for defining patient outcome.

\section{Material and methods}

\section{Patients}

We studied 178 newly diagnosed MM patients enrolled in UK NCRI Myeloma XI (ISRCTN49407852) for which high quality bone marrow tumor material at both presentation and relapse was available. First outcomes of the trial have been published recently [17]. Briefly, patients were initially randomized to triplet induction with thalidomide (CTD), lenalidomide (CRD), or carfilzomib and lenalidomide (KCRD; transplant-eligible patients only) in combination with cyclophosphamide and dexamethasone. Insufficient responders (partial or minimal response) were randomized to cyclophosphamide, bortezomib and dexamethasone (CVD) vs. no intensification and nonresponders (stable or progressive disease) received CVD. Younger, fitter patients received high-dose melphalan (HDMEL) and autologous stem-cell transplantation, patients were randomized to receive lenalidomide, lenalidomide plus vorinostat, or observation. Maintenance treatment continued until progressive disease in the absence of toxicity (Supplementary Fig. 1).

Median time to progression was 20.7 months (range 3.7-71.9 months) and median follow-up 47.0 months. Baseline characteristics are summarized in Supplementary Table 1. The frequency of chromosomal aberrations detectable in the 178 patients at presentation were representative of the overall trial cohort (Supplementary Table 2) [4].

For all patients CD138-positive tumor cells were immunomagnetically selected at presentation and relapse and quality controlled for tumor cell purity (>95\%), DNA and RNA were extracted using QIAGEN (Hilden, Germany) Allprep kits. Additional molecular quality control including longitudinal consistency for $\operatorname{IgD}$ deletion status was performed for all cases. Matched identity of presentation-relapse pairs was confirmed using single nucleotide and/or insertion/deletion polymorphisms assayed by digitalMLPA D006-X2 for all samples.

All patients provided written informed consent. The study was approved by the UK National Research Ethics Service, research ethics committees at participating centers and the UK Medicines and Healthcare Products Regulatory Agency.

\section{Translocation and copy number profiling}

Multiplexed qRT-PCR was used to determine $\mathrm{t}(4 ; 14)$, $\mathrm{t}(6 ; 14), \mathrm{t}(11 ; 14), \mathrm{t}(14 ; 16)$, and $\mathrm{t}(14 ; 20)$ status in tumors, based on the translocation cyclin D (TC) classification system as previously described [5, 18].

Targeted genome wide CNA status at presentation and relapse in each patient was assessed with a newly developed research version of D006-X2 Multiple Myeloma digitalMLPA probemix, as previously described (Supplementary Methods) $[19,20]$. Details of each probe and their respective genomic positions are provided in Supplementary Table 3. CNAs involving sex chromosomes were not considered. CNAs were called if $\geq 50 \%$ of probes mapping to an individual gene or genomic region deviated from normal diploid pattern.

\section{Statistical analyses}

Analyses were performed in $\mathrm{R}$ version 3.5.2 using "dplyr", "tidyr", "stats", "survival" and "survminer", 
"ComplexHeatmap" and "ggpubr" packages. The association between categorical variables was examined using Fishers exact test and between continuous variables using the Wilcoxon signed-rank test. Progression-free survival (PFS) was defined as time from induction randomization to progression, according to International Myeloma Working Group criteria, or death of any cause. Overall survival (OS) was time from induction randomization to death of any cause. Cox proportional hazards $(\mathrm{PH})$ regression was used to estimate univariate and multivariable hazard ratios (HRs) and $95 \%$ confidence intervals (CI). To examine the predictive value of evolution in CNA status for subsequent therapy, high-risk CNA were considered as time dependent covariates within the multivariate model. The PH assumption was tested to investigate whether time dependent covariate effects persisted irrespective of time of acquisition (Supplementary Methods). Multivariate covariates included $\mathrm{t}(4 ; 14), \mathrm{t}(14 ; 16), \operatorname{del}(1 \mathrm{p}), \operatorname{gain}(1 \mathrm{q}), \operatorname{del}(17 \mathrm{p})$ and treatment pathway (transplant eligible vs. not). This is relevant because, although the median follow-up of this cohort is long, the inherent requirement of a relapse biopsy excludes the longest responders from our analyses as they are still on active trial medication or observation with no evidence of disease progression. Differences between Kaplan-Meier survival curves was assessed using the log-rank test. A twosided $P$ value of $\leq 0.05$ was considered statistically significant.

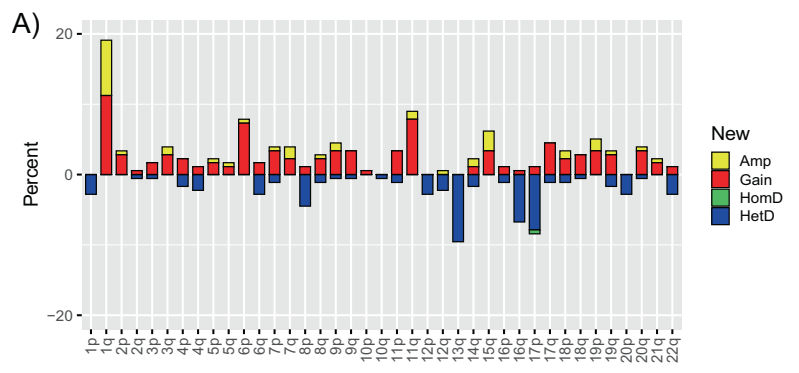

B)

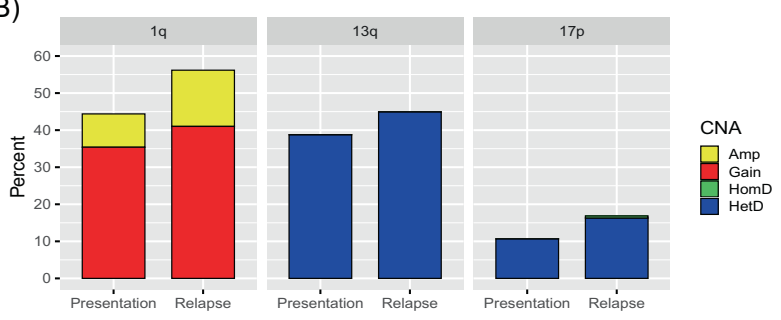

Fig. 1 Recurrent chromosomal and sub-chromosomal CNA changes at relapse. A Frequency of CNAs emerging at relapse affecting chromosome arms, including new gain (red), amplification (yellow), heterozygous deletion (blue), or homozygous deletion (green) plotted as a bar graph. B Bar graph showing frequency of 1q, $13 \mathrm{q}$, and $17 \mathrm{p}$ CNAs, including gain (red), amplification (yellow), heterozygous deletion (blue), or homozygous deletion (green), at presentation and relapse. C Heat map of evolving chromosome 1

\section{Results}

\section{Acquisition of new CNAs is a feature of relapse}

Relapse was associated with the acquisition of new CNAs in $87.1 \%$ of tumors, most $(73.2 \%)$ being large-scale chromosome changes. Across all patients, relapse was associated with a higher number of CNAs: median 11.5 (range $0-34)$ vs. median 12 (range $0-29)(P=0.0058)$. As previously well-documented [16, 21], IGH translocations were clonal at presentation and their status did not change at relapse.

The most frequent chromosomal changes associated with relapse were gain or amplification of 1q (19\%), del(13q) (10\%), gain or amplification of $11 \mathrm{q}(9 \%)$ and $\operatorname{del}(17 \mathrm{p}) / T P 53$ in $(8 \%)$ (Fig. 1; Supplementary Table 2). Although not common, some clonal CNAs detectable at presentation were not detectable at relapse, in particular gain of odd numbered chromosomes in HRD tumors (Supplementary Fig. 2).

\section{Evolution of sub-chromosomal aberrations}

In contrast to 1q gain, which generally involved the whole chromosomal arm, clonal $1 \mathrm{p}$ deletions tended to be subchromosomal (Fig. 1): 1p32.3 deletion (9/178; 5.1\%) implicating $C D K N 2 C$ or 1p12 implicating FAM46C (4/178; $2.2 \%$ ). In nine of 22 tumors with $1 \mathrm{p} 12$ (FAM46C) deletion
C)

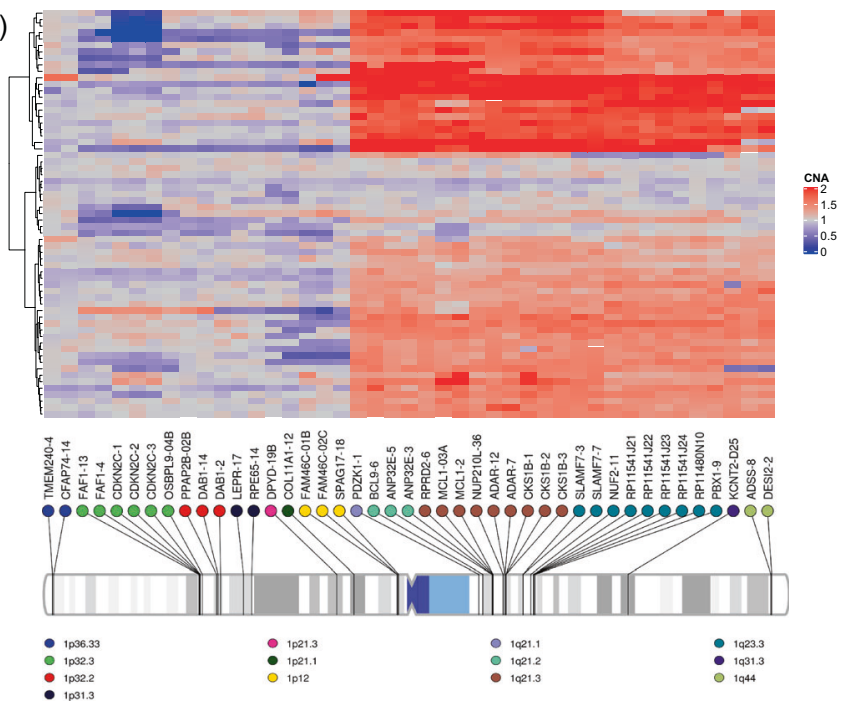

CNAs at relapse, 63 tumors with change in this region at relapse. On the left ( $Y$-axis) dendrogram representing unsupervised clustering analysis of emerging CNAs in areas interrogated by digitalMLPA probes, which are annotated with gene names and chromosomal location below ( $X$-axis). Change per probe and tumor color-coded as per scale representing normalized digitalMLPA copy number ratios with 1.0 equivalent to normal/diploid. 
at diagnosis, the deletion was not clonally detectable at relapse; in contrast all but one of the 8 presentation $1 \mathrm{p} 32.3$ $(C D K N 2 C)$ deletions were detectable at relapse.

Focal gain at relapse also affected 8q24.21, involving the MYC locus, in 10/178 (5.6\%) tumors. In three of 15 tumors with $M Y C$ gain at presentation, reversion to diploid status was a feature of relapse.

Sub-chromosomal CNAs involving IMiD response genes - CRBN (3p26.2), IKZF1 (7p12.2), IKZF3 (17q12), and IRF4 (6p25.3)—were infrequent (Supplementary Fig. 3). Most were detectable in tumors relapsing off therapy (no maintenance); only 2 out of 10 were a feature of tumors from patients in receipt of lenalidomide maintenance (1 deletion of $C R B N ; 1$ gain IRF4).

\section{Evolution of driver copy number aberrations}

We next examined for clonal emergence at relapse of subclonal changes at diagnosis, focusing on the most frequent drivers-gain(1q) and del(17p). We and others showed before that calling of CNAs using conservative high confidence MLPA cutoff values detects clonal infiltration equivalent to about $>20 \%$ by FISH, but that calling of minor sub clones is also feasible [4]. Thirty percent of patients' tumors with clonally detectable 1q gain at relapse had a detectable, potential minor sub-clonal gain(1q) at diagnosis and $50 \%$ of del(17p) relapse tumors had potential subclonal del(17p) at diagnosis (Supplementary Fig. 4). Progressive clonal expansion of $1 \mathrm{q}$ positive tumors was a feature of $17.5 \%$ gain(1q) cases, whereby gain at diagnosis evolved into tetraploidy of 1q (amp(1q)) at relapse. Two of 15 tumors with gain of $M Y C$ at diagnosis progressed to amplification at relapse. This was also a feature with gain of $15 q(4.4 \%)$ and $19 p(2.3 \%)$, albeit at low frequency.

We and others have demonstrated that patients with double or triple hit tumors (i.e., $2+$ high-risk aberrations $t(4 ; 14), t(14 ; 16), t(14 ; 20)$, gain(1q), or del(17p)) have an especially poor prognosis [4]. It was noteworthy that some tumors with 0 or 1 lesions progressed to carrying double, triple and quadruple hits at relapse (Fig. 2). Amplification 1q has recently been proposed as an additional independent marker of high risk by some researchers [22]. In our study around two thirds of $\operatorname{amp}(1 \mathrm{q})$ tumors were "double hit", i.e., also carried $\mathrm{t}(4 ; 14), \mathrm{t}(14 ; 16), \mathrm{t}(14 ; 20)$, or $\operatorname{del}(17 \mathrm{p})$. Of all 178 relapsed tumors, nearly $10 \%$ carried both amp(1q) and "double hit" genetic features (Fig. 2).

\section{Impact of subtype on copy number profile}

Given the biological heterogeneity of MM we examined the relationship between CNAs and disease progression by subtype: those with IG translocations $(\mathrm{t}(4 ; 14), \mathrm{t}(11 ; 14)$, $\mathrm{t}(14 ; 16) / \mathrm{t}(14 ; 20))$, and those with HRD, sub-grouped into those with and without gain of 11, in analogy to Translocation and Cyclin D (TC) classification (Supplementary Table 2) $[4,5]$.

Emerging CNA patterns at relapse were classified as branching ( $45.5 \%$ of all tumors), linear $(22.5 \%)$, linear loss $(19.1 \%)$, and stable (12.9\%) (Fig. 3). In HRD the majority of tumors showed evidence of branching evolution (57.3\%) (Fig. 3). In contrast, linear evolution dominated (36.8\%) $\mathrm{t}(4 ; 14)$ MM. $\mathrm{t}(11 ; 14)$ tumors primarily showed either no CNA change at relapse (stable; 33.3\%), or linear evolution (28.6\%) (Fig. 3). Importantly, there was no relationship between acquisition of CNAs and either HDMEL or lenalidomide maintenance therapy (Fig. 4).

Both del(13) (94.7\%) and gain/amp (1q) (68.4\%) were seen at high frequency in $\mathrm{t}(4 ; 14) \mathrm{MM}$ at presentation, and the CNAs were seen at even higher frequencies in relapse tumors at $100 \%$ and $79.0 \%$, respectively (Supplementary Fig. 5). Moreover, at relapse $\operatorname{del}(17 \mathrm{p})$ was a feature of $31.6 \%$ of $\mathrm{t}(4 ; 14)$ tumors (Supplementary Fig. 5). In contrast, acquisition of $1 \mathrm{q}, 2 \mathrm{p}, 8 \mathrm{q}, 9 \mathrm{p}, 9 \mathrm{q}$, or $6 \mathrm{q}$ CNAs, the changes occurring in $t(11 ; 14)$, were not common; each of these aberration was acquired in $<5 \%$ of $\mathrm{t}(11 ; 14)$ relapsed tumors (Fig. 3). Due to their low frequency, as expected, $\mathrm{t}(14 ; 16)$ (two patients) and $\mathrm{t}(14 ; 20)$ (one patient) tumors are not discussed; sequential CNA analysis providing limited information regarding subgroup evolutionary trajectory. Baseline and relapse CNAs for $t(14 ; 16)$ and $t(14 ; 20)$ shown in Supplementary Fig. 6.

Finally, we examined the relationship between CNA change and MM subtypes defined by $C C N D$ expression; CCND1 (D1), CCND2 (D2), or both (D1 + D2) (Supplementary Fig. 7). Acquisition of $\operatorname{del}(17 \mathrm{p})$ was associated primarily with D2 subtype (Supplementary Fig. 8). Specifically, acquired del(17p) at relapse was a feature of $14.6 \%$ of $\mathrm{D} 2,12.0 \%$ of $\mathrm{D} 1+2$, and $5.1 \%$ of $\mathrm{D} 1$ tumors. Overall, acquisition of new lesions was less frequent in HRD with gain(11), characterized by D1 expression, vs. other HRD tumors (Supplementary Fig. 9).

\section{Associations with patient outcome}

Gain(1q) $(\mathrm{HR}=2.23 ; P<0.001), \operatorname{del}(1 \mathrm{p}) / C D K N 2 C(\mathrm{HR}=$ $1.81 ; P=0.046)$, gain $(8 \mathrm{q}) / M Y C(\mathrm{HR}=1.87 ; P=0.033)$, and $\operatorname{del}(17 \mathrm{p})(\mathrm{HR}=2.95 ; P<0.001)$ considered as time dependent variables, i.e., including their acquisition at relapse, were all independently associated with shorter OS (Table 1; Supplementary Fig. 10; Supplementary Table 4).

CNAs were stratified by time point of acquisition, i.e., "Gain-Gain" for those with stable gain and "Diploid-Gain" for those with evolution of gain(1q) at relapse. Gain(1q) from presentation and evolution of new gain(1q) at relapse were both associated with significantly shorter OS compared to normal 1q copy number (HR 2.11; $P=0.0040$ and 
A)

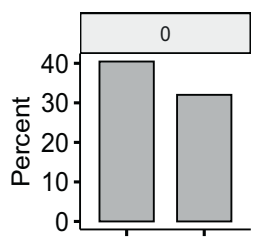

High Risk Lesions $\square 0 \square 1 \square 2 \square 3 \square 4$
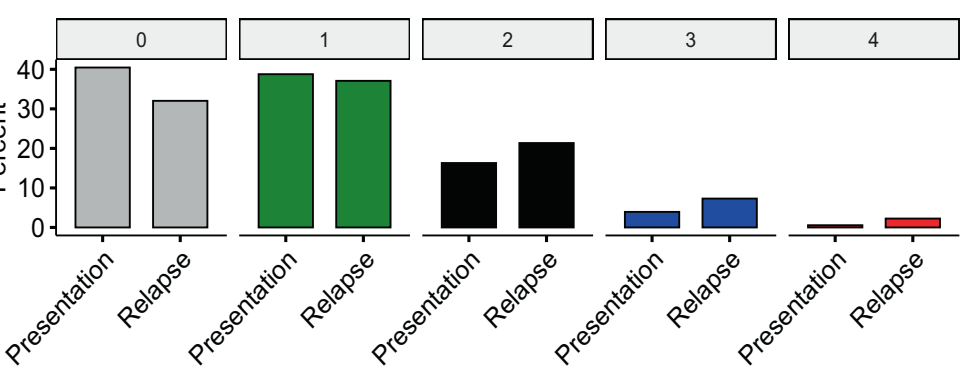

Presentation

B)

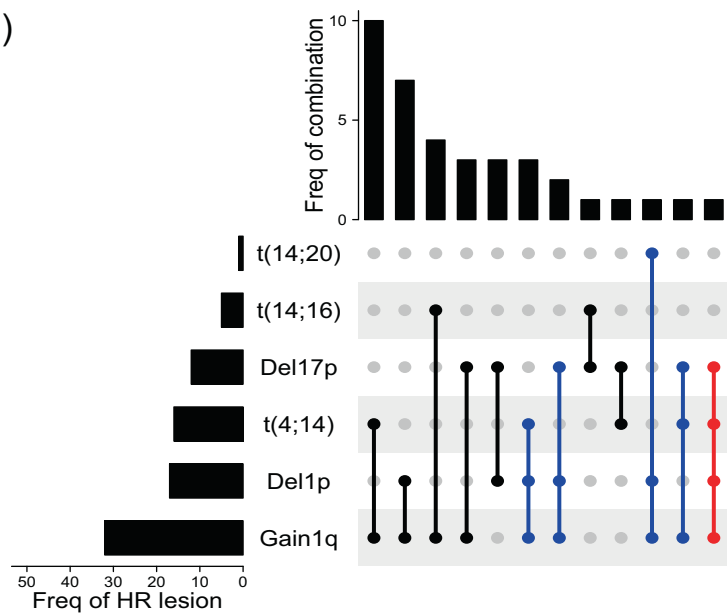

Fig. 2 Positive selection of co-occurrence of high-risk lesions. A Frequency bar chart of tumors characterized by number of high-risk markers, ranging from no (0) high-risk marker to co-occurrence of 4 markers. B Upset plots of Presentation (left) and Relapse (right) tumors, each showing frequency of individual high-risk lesions (left), type of combination of lesions (center lines with dots indicating

HR $2.00 ; P=0.021$, respectively). Median OS was 44.3 vs. 47.9 vs. 67.1 months for gain(1q) at diagnosis, new gain (1q) at relapse and normal 1q copy number at both time points, respectively (log-rank $P=0.007$ ) (Fig. 5).

Acquired $\operatorname{del}(17 \mathrm{p}), \operatorname{del}(1 \mathrm{p} / C D K N 2 C)$ or gain $(8 \mathrm{q} / M Y C)$ at relapse were not clearly associated with a significantly worse OS in subgroup analyses, although our power to demonstrate a relationship was naturally limited by low frequency of these CNAs. Median OS was 31.3 vs. 60.5 vs. 65.4 months for del(17p) at diagnosis, emergence at relapse and absence at both, respectively (log-rank $P<0.001$ ) (Fig. 5). Median OS was 38.3 vs. 44.6 vs. 63.4 months for $\operatorname{del}(1 \mathrm{p} / C D K N 2 C)$ at diagnosis, emergence at relapse or absence, respectively (log-rank $P=0.03$ ) (Fig. 5); and for gain $(8 \mathrm{q} / M Y C), 38.3$ vs. 36.2 vs. 63.4 months, respectively (log-rank $P=0.07$ ) (Fig. 5).

Of interest, type of evolutionary pattern was associated with OS; branching evolution was significantly associated with the shortest and linear loss with the longest OS (HR

\begin{tabular}{cccc}
40 & 30 & 20 & 10 \\
\hline
\end{tabular}
C)

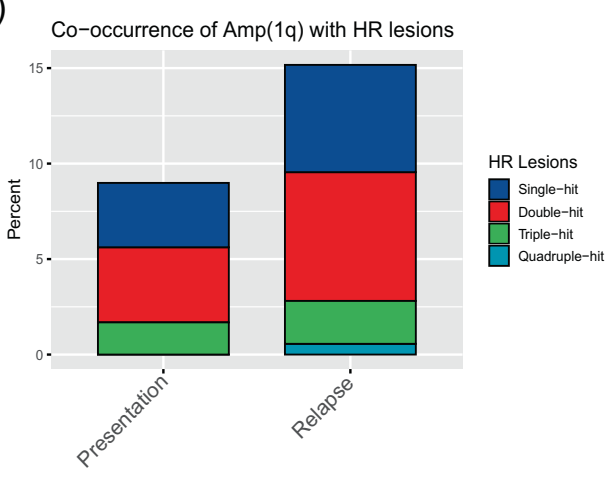

Relapse
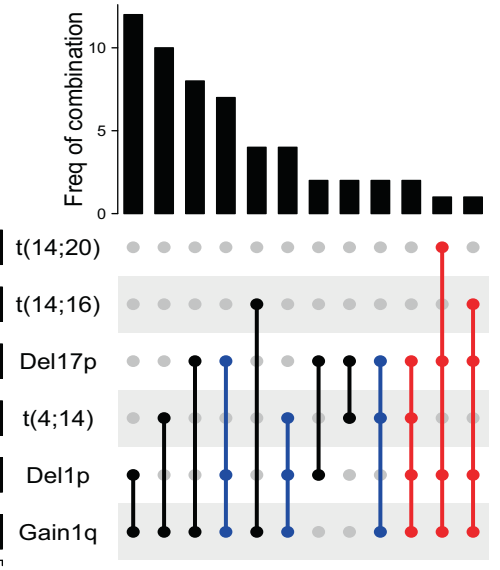

presence of individual lesion) and frequency of specific combination of markers (top). C Bar chart showing the overall frequency of amp(1q) between presentation and relapse (overall bar height) and proportion of $\operatorname{amp}(1 \mathrm{q})$ tumors showing $1 \mathrm{q}$ as the only high-risk aberration (single hit), or in combination with one or more other high-risk markers (double hit to quadruple hit).

$2.61, P=0.0048$ ), with median OS being 44.6 vs. 59.6 vs. 62.0 vs. 68.1 months for branching, linear, no change and linear loss respectively (log-rank $P=0.02)$ (Fig. 5).

\section{Discussion}

Our analysis demonstrates that progression of $\mathrm{MM}$ is characterized by emergence of clones with additional largescale chromosomal changes, commonly involving 1q. Gain or amp(1q) frequently co-evolves with other CNAs, either implicating 1q directly in their genesis as per "jumping 1q" hypothesis, or highlighting 1q as a region providing clonal advantage for genetically instable tumors [23]. While a number of genes on 1q have been proposed as drivers, our study does not provide data to make a specific inference $[8,24-27]$. Our findings also suggest progression of $\mathrm{MM}$ is associated with positive selection of sub-chromosomal loss of $1 \mathrm{p}$, implicating $C D K N 2 C$, and thereby indirectly 


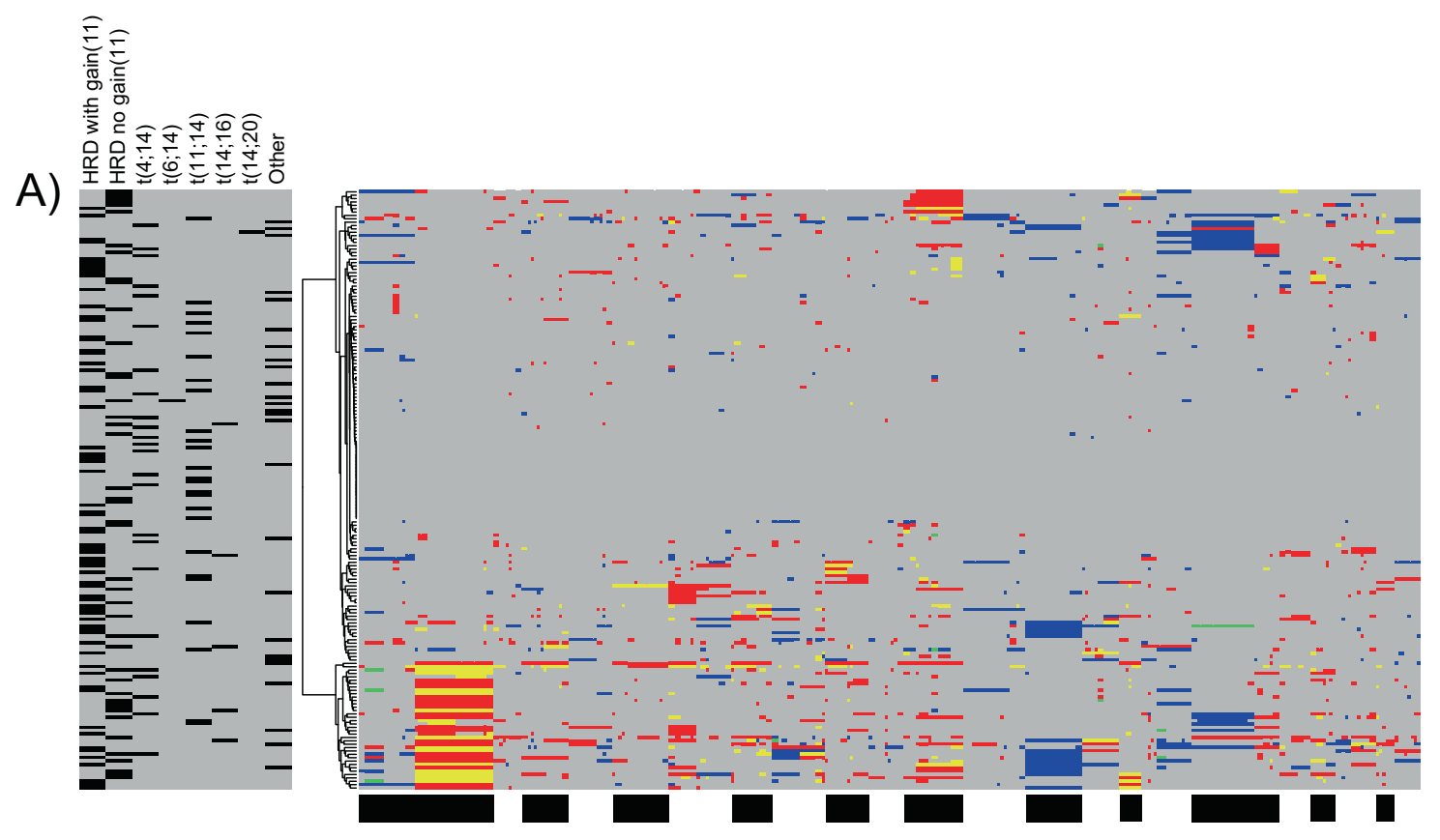

B)

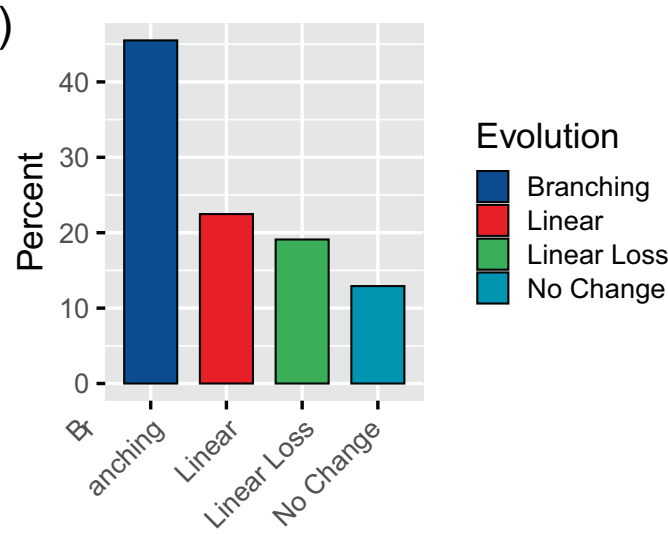

C)

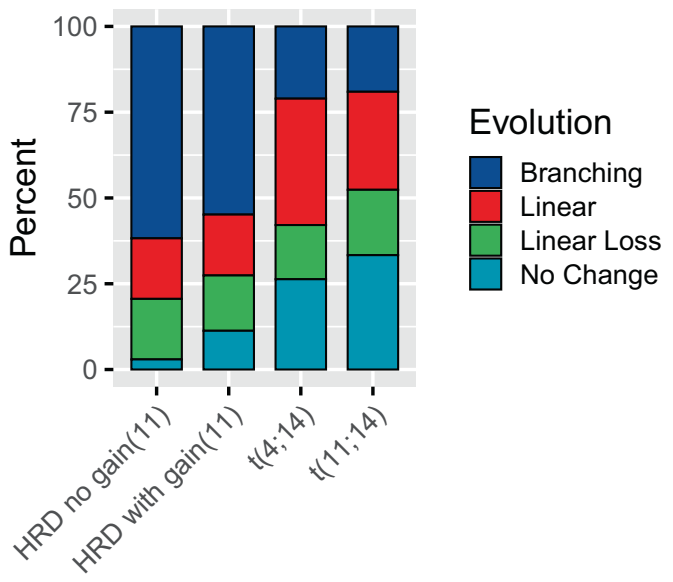

right in ascending order of genomic position) displayed in a heat map with unsupervised clustering for evolving CNAs. B Frequency of evolutionary patterns across all tumors. C Frequency of evolutionary patterns per major molecular MM subgroups.

evolution of pre-therapeutic mutational patterns but extend these in context of therapy and relapse [30-32]. We also describe, to our knowledge for the first time, an association between CNA evolution pattern and subsequent outcome. Whether individual CNA evolution pattern has independent clinical relevance and could be included in management considerations at relapse requires further investigation.

We identified only a low frequency of CNAs to which IMiD response genes map. In conjuncture with published somatic SNV data, results suggest mutation of these genes per se is not the major determinant of acquired resistance to lenalidomide, or other members of this class of agent in MM [12, 14-16, 33-35]. 
A)

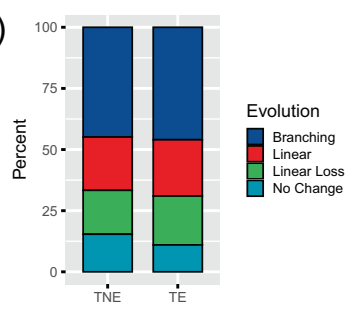

B)

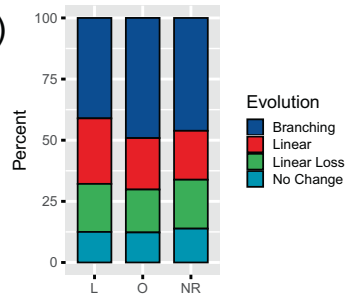

C)

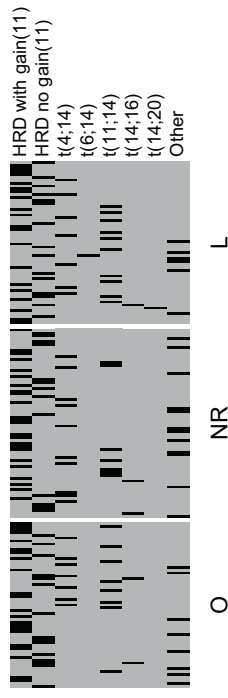

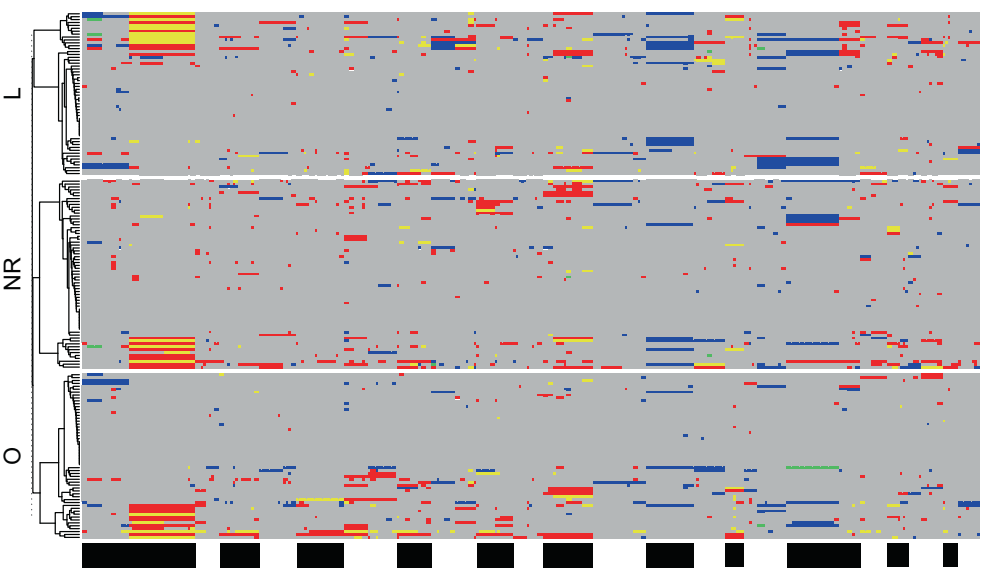

Fig. 4 Evolutionary patterns in context of trial treatment. A Comparison of frequencies of evolutionary patterns between transplant eligible (TE; younger, fitter patients) and transplant non eligible (TNE). B Comparison of frequencies of evolutionary patterns for patients randomized to lenalidomide (L) maintenance, observation $(\mathrm{O})$ or those not randomized (NR) (C) Heatmaps of CNA changes at relapse for tumors from patients randomized to lenalidomide (L) maintenance, observation $(\mathrm{O})$ or those not randomized (NR). For each group separately unsupervised clustering on CNA changes was performed and a dendrogram is shown on the left of each heat map. Legend on left side provides context on molecular background lesions $($ dark $b a r=$ present, gray $=$ absent $)$. Legend at bottom: black and white bands representing chromosomal mapping of digitalMLPA probes chr1-22 from left to right in ascending order of genomic position.
Table 1 Multivariate Cox regression analysis of risk factors for OS, where CNAs represent time dependent covariates.

\begin{tabular}{llll}
\hline & HR & $95 \%$ CI & $P$ value \\
\hline $\mathrm{t}(4 ; 14)$ & 1.25 & $(0.62-2.54)$ & 0.53 \\
$\mathrm{t}(14 ; 16)$ & 1.75 & $(0.66-4.60)$ & 0.26 \\
Gain(1q) & 2.23 & $(1.39-3.57)$ & 0.0008 \\
Del(1p)/CDKN2C & 1.81 & $(1.01-2.26)$ & 0.046 \\
Gain(8q)/MYC & 1.87 & $(1.05-3.31)$ & 0.033 \\
Del(17p) & 2.95 & $(1.67-5.20)$ & 0.0001 \\
TNE & 2.30 & $(1.41-3.75)$ & 0.0008 \\
\hline
\end{tabular}

$H R$ hazard ratio, $95 \%$ CI 95\% confidence interval.

We demonstrate, to our knowledge for the first time, in a randomized controlled trial that acquisition of gain(1q) at relapse is independently and strongly associated with inferior OS [36]. Although findings are also indicative for $\operatorname{del}(17 \mathrm{p})$, del(1p/CDKN2C) and gain(8q/MYC), our power to robustly assert clinical relevance of acquired lesions at relapse is inevitably limited by the lower frequency of these CNAs [37]. Clinical evaluation of these markers at relapse is technically feasible in most developed healthcare systems, but currently not widely recommended [38-40].

It is unknown whether sub-clones residing outside of the standard iliac crest bone marrow sampling area at diagnosis are the main source of clonal evolution detected at relapse or whether these predominantly emerge through ongoing genomic instability [12]. Multi-region bone sampling in MM is associated with significant risk and morbidity, making its implementation as part of standard care very challenging. Functional bone marrow soft tissue imaging techniques such as diffusion-weighted whole body MRI and/or molecular techniques including circulating tumor DNA profiling are promising methods in development which may contribute to diagnostic assessment of spatial MM heterogeneity in the future. However, sensitivity, precision and clinical relevance of minor sub-clone detection from a single time point for these methods remains to be established, in particular if results differ from those of parallel bone marrow sampling, before informing clinical management [41-44].

All patients received uniform trial treatment, in particular providing insight into thalidomide and lenalidomide associated CNA changes. However, patients also received lowdoses of the oral alkylator cyclophosphamide during induction, which may have specific impact on CNA changes, potentially limiting generalizability of our findings.

In addition, molecular information generated with a targeted tool like digitalMLPA is inherently focused and does not discriminate complex processes potentially underlying identified CNAs, such as chromotripsis or chromoplexy, which are detectable by discovery tools such as whole genome sequencing, or single nucleotide variants captured by myeloma specific CNA/sequencing panels [29, 45-47]. Our data also cannot reflect accompanying changes of the tumor microenvironment, which have been implicated in myeloma progression [48]. However, digitalMLPA is in development for clinical diagnostic application, offers sensitive CNA 


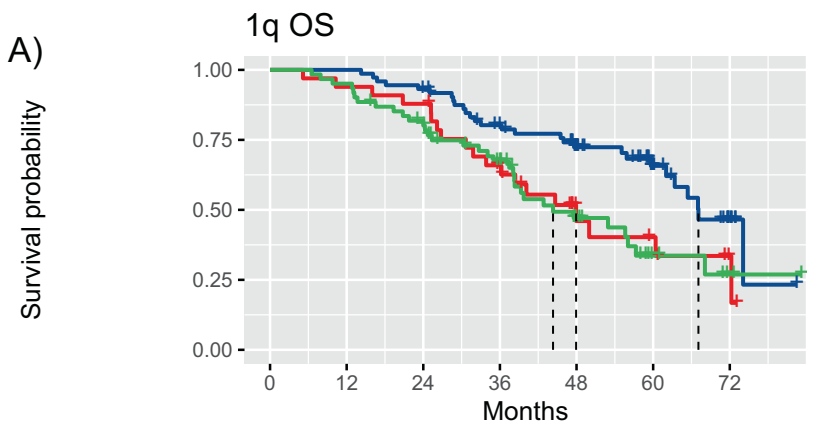

Number at risk

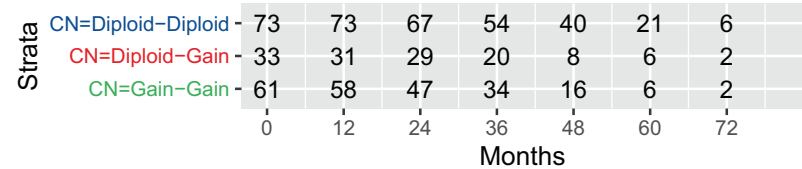

C)

$$
17 p \text { OS }
$$
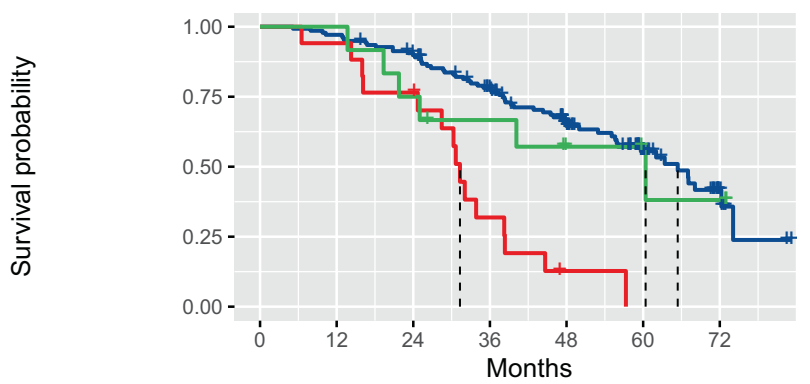

Number at risk

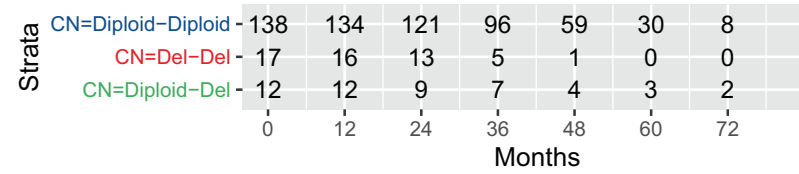

E)

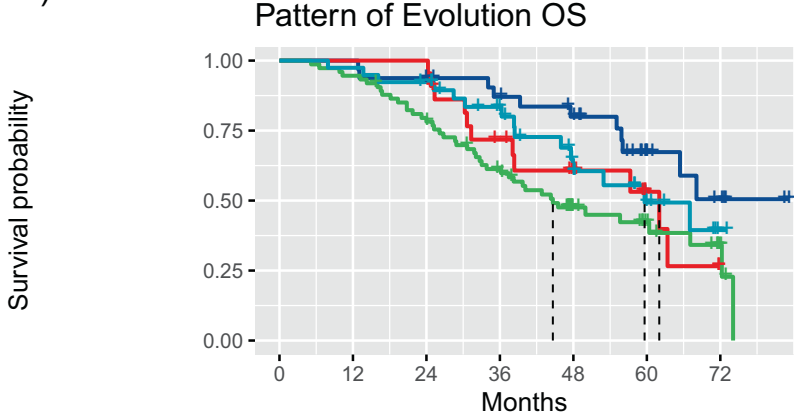

Number at risk

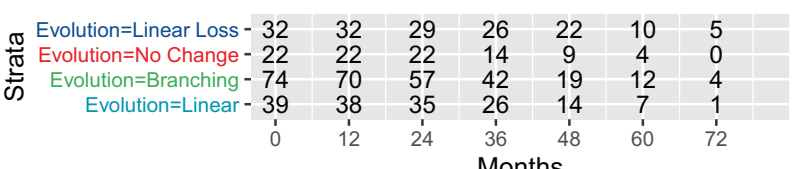

Fig. 5 Relationship between emerging CNAs, evolutionary trajectories, and patient outcome. A Kaplan-Meier plot for overall survival in context of presence of CNAs for 1q. Equivalent plots for aberrations of (B) deletion of $1 \mathrm{p} 21(C D K N 2 C)$ (C) gain of $8 \mathrm{q} 21$

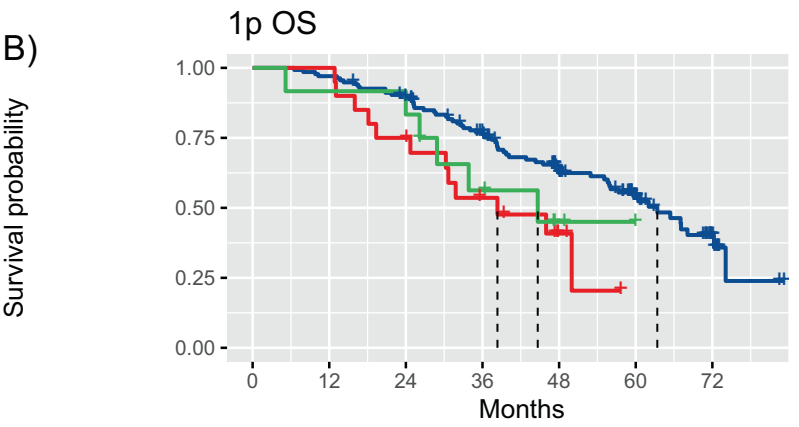

Number at risk

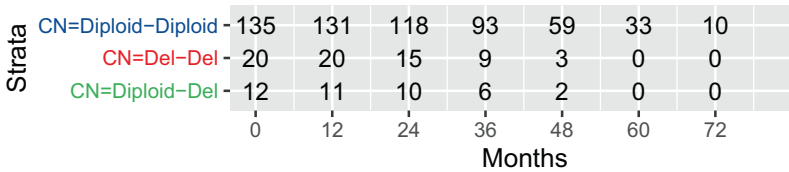

D)

\section{MYC OS}

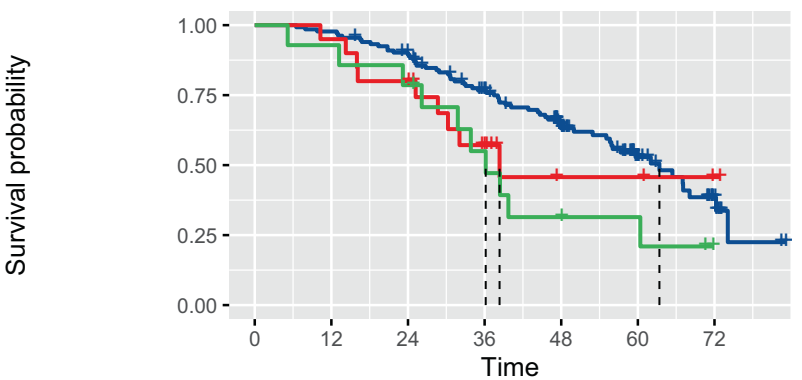

Number at risk

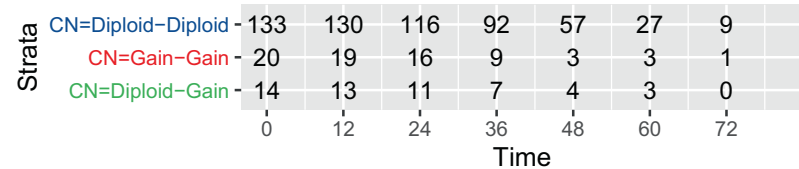

(MYC) (D) deletion of 17p (TP53) (E) evolutionary pattern. As per individual color-coded label for $\mathbf{A}-\mathbf{D}$, curves represent patients with tumors remaining diploid at both time points, changing from diploid to abnormal or showing abnormality at both time points. 
information specific to myeloma from low tumor DNA quantities at high throughput. It requires limited computational infrastructure and uses standardized analysis algorithms, thus overcoming the significant limitations of conventional FISH analysis [20]. As our study demonstrates, digitalMLPA is suitable for longitudinal intra-individual tracking and provides a standardized and accessible method for cross-study validation and putative implementation in molecularly stratified prospective clinical trials.

Genetic re-profiling at relapse in MM is currently not widely recommended and, as a consequence, often not reimbursed [10, 39, 49]. Whilst treatment options for relapsed disease were until recently very limited, a range of therapeutic regimens with varying intensity are now approved and available, requiring better tools for clinical decision making at relapse [50-53]. Adapting first-line therapy and its intensity to individual tumor risk markers in MM is now a key focus of research, with multiple genetically stratified prospective clinical trials currently ongoing $[10,54,55]$. The debate about optimal tailoring of second line treatment is likely to intensify with increasing therapeutic options. Our study demonstrates not only the frequency of emerging high-risk CNAs at MM relapse, but also the unmet clinical need of patients with chromosomally evolving MM. These patients should ideally be recognized early during relapse and prospective clinical trials investigating longitudinal management strategies adapted to dynamic risk profiling, designed [56].

Our findings strongly support repeated tumor molecular analysis in MM in context of modern treatment, even in circumstances where only selected markers such as $1 \mathrm{q}$ can be tested, as a means of tailoring patient treatment beyond first-line therapy.

Acknowledgements This work was supported by research grants from Celgene, Myeloma UK and the National Institute of Health Biomedical Research Center at the Royal Marsden Hospital and Institute of Cancer Research, London. MFK was supported by a Jacquelin ForbesNixon Fellowship. Primary financial support for NCRI Myeloma XI was provided by Cancer Research UK (C1298/A10410). Unrestricted educational grants from Novartis, Schering Health Care, Chugai, Pharmion, Celgene, Ortho Biotech, Amgen, and Merck Sharp \& Dohme supported trial coordination. We are grateful for the support of the Clinical Trials Research Unit (CTRU) at Leeds, to the NCRI Haemato-oncology subgroup, investigators and patients. The Myeloma XI trial was conducted with support of the NCRI Hematological Oncology Clinical Studies Group.

Author contributions Conception and design: JC, RSH, and MFK. Acquisition of data: all authors. Analysis of data: JC, MFK. Paper writing: JC, RSH, and MFK.

\section{Compliance with ethical standards}

Conflict of interest MWJ: Janssen-consultancy, honoraria, travel support, research funding; Takeda-consultancy, honoraria, travel support; Amgen-consultancy, honoraria, travel support; Celgene Corporation-consultancy, honoraria, research funding; Novartisconsultancy, honoraria. MTD: Abingdon Health-equity ownership, membership on an entity's board of directors or advisory committees. RGO: Takeda-honoraria, travel support; Janssen-consultancy, travel support; Celgene Corporation-consultancy, honoraria, research funding. JL: Janssen-consultancy; Novartis—travel support; Takeda - honoraria, travel support; Bristol-Myers Squibb—consultancy, travel support; Celgene Corporation-consultancy, honoraria, travel support. KK: Celgene Corporation-travel support, research funding; Janssen - travel support, research funding. WMG: Celgene Corporation-consultancy, research funding; Amgen, Merck Sharp and Dohme-research funding; Janssen-honoraria. FED: Amgen - consultancy, honoraria; AbbVie - consultancy, honoraria; Takeda-consultancy, honoraria; Janssen-consultancy, honoraria; Celgene Corporation-consultancy, honoraria, research funding; Roche-consultancy, honoraria. GJM: Janssen-research funding; Bristol-Myers Squibb-consultancy, honoraria; Takeda - consultancy, honoraria; Celgene Corporation-consultancy, honoraria, research funding; Roche-consultancy, honoraria; Amgen-consultancy, honoraria; GSK - consultancy, honoraria; Karyopharm - consultancy, honoraria. GC: Takeda-consultancy, honoraria, research funding, speakers bureau; Glycomimetics-consultancy, honoraria; Sanofi-consultancy, honoraria, speakers bureau; Celgene Corporation-consultancy, honoraria, research funding, speakers bureau; Janssenconsultancy, honoraria, research funding, speakers bureau; BristolMyers Squibb - consultancy, honoraria; Amgen-consultancy, honoraria, research funding, speakers bureau. LA: MRC-Hollandemployment. SS: MRC-Holland-employment. DAC: Celgene Corporation, Amgen, Merck Sharp and Dohme-research funding. GHJ: Roche-consultancy, honoraria, speakers bureau; Amgen-consultancy, honoraria, speakers bureau; Janssen-consultancy, honoraria, speakers bureau; Merck Sharp and Dohme-consultancy, honoraria, speakers bureau; Celgene Corporation-consultancy, honoraria, travel support, research funding, speakers bureau; Takedaconsultancy, honoraria, travel support, research funding, speakers bureau. MFK: AbbVie-consultancy; Bristol-Myers Squibb-consultancy, travel support; Chugai-consultancy; GSK-consultancy; Janssen-consultancy, honoraria, research funding; Amgen-consultancy, honoraria; Takeda-consultancy, travel support; Celgene Corporation-consultancy, honoraria, and research funding.

Publisher's note Springer Nature remains neutral with regard to jurisdictional claims in published maps and institutional affiliations.

Open Access This article is licensed under a Creative Commons Attribution 4.0 International License, which permits use, sharing, adaptation, distribution and reproduction in any medium or format, as long as you give appropriate credit to the original author(s) and the source, provide a link to the Creative Commons license, and indicate if changes were made. The images or other third party material in this article are included in the article's Creative Commons license, unless indicated otherwise in a credit line to the material. If material is not included in the article's Creative Commons license and your intended use is not permitted by statutory regulation or exceeds the permitted use, you will need to obtain permission directly from the copyright holder. To view a copy of this license, visit http://creativecommons. org/licenses/by/4.0/.

\section{References}

1. Maura F, Bolli N, Rustad EH, Hultcrantz M, Munshi N, Landgren $\mathrm{O}$. Moving from cancer burden to cancer genomics for smoldering myeloma: a review. JAMA Oncol. 2020;6:425-32. 
2. Manier S, Salem KZ, Park J, Landau DA, Getz G, Ghobrial IM. Genomic complexity of multiple myeloma and its clinical implications. Nat Rev Clin Oncol. 2017;14:100-13.

3. Morgan GJ, Walker BA, Davies FE. The genetic architecture of multiple myeloma. Nat Rev Cancer. 2012;12:335-48.

4. Shah V, Sherborne AL, Walker BA, Johnson DC, Boyle EM, Ellis $\mathrm{S}$, et al. Prediction of outcome in newly diagnosed myeloma: a meta-analysis of the molecular profiles of 1905 trial patients. Leukemia. 2018;32:102-10.

5. Bergsagel PL, Kuehl WM, Zhan F, Sawyer J, Barlogie B, Shaughnessy J. Cyclin D dysregulation: an early and unifying pathogenic event in multiple myeloma. Blood. 2005;106:296-303.

6. Boyd KD, Ross FM, Chiecchio L, Dagrada GP, Konn ZJ, Tapper WJ, et al. A novel prognostic model in myeloma based on cosegregating adverse FISH lesions and the ISS: analysis of patients treated in the MRC Myeloma IX trial. Leukemia. 2012;26:349-55.

7. Perrot A, Lauwers-Cances V, Tournay E, Hulin C, Chretien M-L, Royer B, et al. Development and validation of a cytogenetic prognostic index predicting survival in multiple myeloma. J Clin Oncol. 2019;37:1657-65.

8. Schmidt TM, Barwick BG, Joseph N, Heffner LT, Hofmeister CC, Bernal L, et al. Gain of Chromosome 1q is associated with early progression in multiple myeloma patients treated with lenalidomide, bortezomib, and dexamethasone. Blood Cancer J. 2019;9:94.

9. Caers J, Garderet L, Kortum KM, O’Dwyer ME, van de Donk N, Binder M, et al. European Myeloma Network recommendations on tools for the diagnosis and monitoring of multiple myeloma: what to use and when. Haematologica. 2018;103:1772-84.

10. Sonneveld P, Avet-Loiseau H, Lonial S, Usmani S, Siegel D, Anderson KC, et al. Treatment of multiple myeloma with highrisk cytogenetics: a consensus of the International Myeloma Working Group. Blood. 2016;127:2955-62.

11. Keats JJ, Chesi M, Egan JB, Garbitt VM, Palmer SE, Braggio E, et al. Clonal competition with alternating dominance in multiple myeloma. Blood. 2012;120:1067-76.

12. Weinhold N, Ashby C, Rasche L, Chavan SS, Stein C, Stephens $\mathrm{OW}$, et al. Clonal selection and double-hit events involving tumor suppressor genes underlie relapse in myeloma. Blood. 2016;128:1735-44

13. Corre J, Cleynen A, Robiou du Pont S, Buisson L, Bolli N, Attal $\mathrm{M}$, et al. Multiple myeloma clonal evolution in homogeneously treated patients. Leukemia. 2018;32:2636-47.

14. Jones JR, Weinhold N, Ashby C, Walker BA, Wardell C, Pawlyn $\mathrm{C}$, et al. Clonal evolution in myeloma: the impact of maintenance lenalidomide and depth of response on the genetics and sub-clonal structure of relapsed disease in uniformly treated newly diagnosed patients. Haematologica. 2019;104:1440-50.

15. Kortum KM, Mai EK, Hanafiah NH, Shi CX, Zhu YX, Bruins L, et al. Targeted sequencing of refractory myeloma reveals a high incidence of mutations in CRBN and Ras pathway genes. Blood. 2016;128:1226-33.

16. Corre J, Cleynen A, Robiou du Pont S, Buisson L, Bolli N, Attal $\mathrm{M}$, et al. Multiple myeloma clonal evolution in homogeneously treated patients. Leukemia. 2018;32:2636-47.

17. Jackson GH, Davies FE, Pawlyn C, Cairns DA, Striha A, Collett $\mathrm{C}$, et al. Lenalidomide maintenance versus observation for patients with newly diagnosed multiple myeloma (Myeloma XI): a multicentre, open-label, randomised, phase 3 trial. Lancet Oncol. 2019;20:57-73.

18. Kaiser MF, Walker BA, Hockley SL, Begum DB, Wardell CP, Gonzalez D, et al. A TC classification-based predictor for multiple myeloma using multiplexed real-time quantitative PCR. Leukemia. 2013;27:1754-7.
19. Benard-Slagter A, Zondervan I, de Groot K, Ghazavi F, Sarhadi V, Van Vlierberghe P, et al. Digital multiplex ligation-dependent probe amplification for detection of key copy number alterations in $\mathrm{T}$ - and B-cell lymphoblastic leukemia. J Mol Diagn. 2017;19:659-72.

20. Kosztolányi S, Kiss R, Atanesyan L, Gángó A, de Groot K, Steenkamer M, et al. High-throughput copy number profiling by digital multiplex ligation-dependent probe amplification in multiple myeloma. J Mol Diagn. 2018;20:777-88.

21. Maura F, Bolli N, Angelopoulos N, Dawson KJ, Leongamornlert D, Martincorena I, et al. Genomic landscape and chronological reconstruction of driver events in multiple myeloma. Nat Commun. 2019;10:3835.

22. Walker BA, Mavrommatis K, Wardell CP, Ashby TC, Bauer M, Davies F, et al. A high-risk, double-hit, group of newly diagnosed myeloma identified by genomic analysis. Leukemia. 2019;33:159-70.

23. Sawyer JR, Tian E, Walker BA, Wardell C, Lukacs JL, Sammartino $\mathrm{G}$, et al. An acquired high-risk chromosome instability phenotype in multiple myeloma: Jumping 1q syndrome. Blood Cancer J. 2019;9:62.

24. Lazzari E, Mondala PK, Santos ND, Miller AC, Pineda G, Jiang $\mathrm{Q}$, et al. Alu-dependent RNA editing of GLI1 promotes malignant regeneration in multiple myeloma. Nat Commun. 2017;8:1922.

25. Marchesini M, Ogoti Y, Fiorini E, Samur AA, Nezi L, D'Anca M, et al. ILF2 is a regulator of RNA splicing and DNA damage response in 1q21-amplified multiple myeloma. Cancer Cell. 2017;32:88.

26. Walker BA, Leone PE, Chiecchio L, Dickens NJ, Jenner MW, Boyd KD, et al. A compendium of myeloma-associated chromosomal copy number abnormalities and their prognostic value. Blood. 2010;116:E56-65.

27. Slomp A, Moesbergen LM, Gong J-N, Cuenca M, von dem Borne PA, Sonneveld P, et al. Multiple myeloma with 1q21 amplification is highly sensitive to MCL-1 targeting. Blood Adv. 2019;3:4202-14

28. Calado DP, Sasaki Y, Godinho SA, Pellerin A, Kochert K, Sleckman BP, et al. The cell-cycle regulator c-Myc is essential for the formation and maintenance of germinal centers. Nat Immunol. 2012;13:1092-100.

29. Hoang PH, Dobbins SE, Cornish AJ, Chubb D, Law PJ, Kaiser M, et al. Whole-genome sequencing of multiple myeloma reveals oncogenic pathways are targeted somatically through multiple mechanisms. Leukemia. 2018;32:2459-70.

30. Johnson DC, Lenive O, Mitchell J, Jackson G, Owen R, Drayson $\mathrm{M}$, et al. Neutral tumor evolution in myeloma is associated with poor prognosis. Blood. 2017;130:1639-43.

31. Bolli N, Maura F, Minvielle S, Gloznik D, Szalat R, Fullam A, et al. Genomic patterns of progression in smoldering multiple myeloma. Nat Commun. 2018;9:3363.

32. Walker BA, Wardell CP, Murison A, Boyle EM, Begum DB, Dahir NM, et al. APOBEC family mutational signatures are associated with poor prognosis translocations in multiple myeloma. Nat Commun. 2015;6:6997.

33. Parzych K, Saavedra-García P, Valbuena GN, Al-Sadah HA, Robinson ME, Penfold L, et al. The coordinated action of VCP/p97 and GCN2 regulates cancer cell metabolism and proteostasis during nutrient limitation. Oncogene. 2019;38:3216-31.

34. Ravi P, Kumar SK, Cerhan JR, Maurer MJ, Dingli D, Ansell SM, et al. Defining cure in multiple myeloma: a comparative study of outcomes of young individuals with myeloma and curable hematologic malignancies. Blood Cancer J. 2018;8:26.

35. Flores A, Sandoval-Gonzalez S, Takahashi R, Krall A, Sathe L, Wei L, et al. Increased lactate dehydrogenase activity is dispensable in squamous carcinoma cells of origin. Nat Commun. 2019;10:91. 
36. Corre J, Montes L, Martin E, Perrot A, Caillot D, Leleu X, et al. Early relapse after autologous transplant for myeloma is associated with poor survival regardless of cytogenetic risk. Haematologica. 2019;105:236588.

37. Shah V, Johnson DC, Sherborne AL, Ellis S, Aldridge FM, Howard-Reeves J, et al. Subclonal TP53 copy number is associated with prognosis in multiple myeloma. Blood. 2018;132:2465-9.

38. Chng WJ, Dispenzieri A, Chim CS, Fonseca R, Goldschmidt H, Lentzsch S, et al. IMWG consensus on risk stratification in multiple myeloma. Leukemia. 2014;28:269-77.

39. NICE Guideline on Myeloma: diagnosis and management. 2020. https://www.nice.org.uk/guidance/ng35.

40. Palumbo A, Avet-Loiseau H, Oliva S, Lokhorst HM, Goldschmidt $\mathrm{H}$, Rosinol L, et al. Revised international staging system for multiple myeloma: a report from international myeloma working group. J Clin Oncol. 2015;33:2863-9.

41. Messiou C, Kaiser M. Whole body diffusion weighted MRI-a new view of myeloma. Br J Haematol. 2015;171:29-37.

42. Messiou C, Hillengass J, Delorme S, Lecouvet FE, Moulopoulos LA, Collins DJ, et al. Guidelines for acquisition, interpretation, and reporting of whole-body MRI in myeloma: myeloma response assessment and diagnosis system (MY-RADS). Radiology. 2019;291:5-13.

43. Guo G, Raje NS, Seifer C, Kloeber J, Isenhart R, Ha G, et al. Genomic discovery and clonal tracking in multiple myeloma by cellfree DNA sequencing. Leukemia. 2018;32:1838-41.

44. Waldschmidt JM, Vijaykumar T, Knoechel B, Lohr JG. Tracking myeloma tumor DNA in peripheral blood. Best Pract Res Clin Haematol. 2020;33:101146.

45. Yellapantula V, Hultcrantz M, Rustad EH, Wasserman E, Londono D, Cimera R, et al. Comprehensive detection of recurring genomic abnormalities: a targeted sequencing approach for multiple myeloma. Blood Cancer J. 2019;9:101.

46. Maura F, Rustad EH, Yellapantula V, Łuksza M, Hoyos D, Maclachlan $\mathrm{KH}$, et al. Role of AID in the temporal pattern of acquisition of driver mutations in multiple myeloma. Leukemia. 2020;34:1476-80.

47. Lawson MA, McDonald MM, Kovacic N, Hua Khoo W, Terry RL, Down J, et al. Osteoclasts control reactivation of dormant myeloma cells by remodelling the endosteal niche. Nat Commun. 2015;6:8983

48. Zavidij O, Haradhvala NJ, Mouhieddine TH, SklavenitisPistofidis R, Cai S, Reidy M, et al. Single-cell RNA sequencing reveals compromised immune microenvironment in precursor stages of multiple myeloma. Nat Cancer. 2020;1:493-506.

49. Mikhael JR, Dingli D, Roy V, Reeder CB, Buadi FK, Hayman $\mathrm{SR}$, et al. Management of newly diagnosed symptomatic multiple myeloma: updated Mayo Stratification of Myeloma and RiskAdapted Therapy (mSMART) consensus guidelines 2013. Mayo Clin Proc. 2013;88:360-76.

50. Garderet L, Kuhnowski F, Berge B, Roussel M, Escoffre-Barbe M, Lafon I, et al. Pomalidomide, cyclophosphamide, and dexamethasone for relapsed multiple myeloma. Blood. 2018;132:2555-63.

51. Bahlis NJ, Dimopoulos MA, White DJ, Benboubker L, Cook G, Leiba $M$, et al. Daratumumab plus lenalidomide and dexamethasone in relapsed/refractory multiple myeloma: extended follow-up of POLLUX, a randomized, open-label, phase 3 study. Leukemia. 2020;34:1875-84.

52. Hari P, Mateos MV, Abonour R, Knop S, Bensinger W, Ludwig H, et al. Efficacy and safety of carfilzomib regimens in multiple myeloma patients relapsing after autologous stem cell transplant: ASPIRE and ENDEAVOR outcomes. Leukemia. 2017;31:2630-41.

53. Palumbo A, Chanan-Khan A, Weisel K, Nooka AK, Masszi T, Beksac M, et al. Daratumumab, bortezomib, and dexamethasone for multiple myeloma. N Engl J Med. 2016;375:754-66.

54. Jenner M, Sherborne AL, Hall A, Shah V, Walker K, Ellis S, et al. Molecular treatment stratification for newly diagnosed high-risk myeloma, including plasma cell leukemia-feasibility results of the Ukmra Optimum: MUK9 Trial (NCT03188172). Blood. 2019;134 Supplement_1:3162.

55. Joseph NS, Kaufman JL, Dhodapkar MV, Hofmeister CC, Almaula DK, Heffner LT, et al. Long-term follow-up results of lenalidomide, bortezomib, and dexamethasone induction therapy and risk-adapted maintenance approach in newly diagnosed multiple myeloma. J Clin Oncol. 2020;38:1928-37.

56. Kurtz DM, Esfahani MS, Scherer F, Soo J, Jin MC, Liu CL, et al. Dynamic risk profiling using serial tumor biomarkers for personalized outcome prediction. Cell. 2019;178:699-713.e19. 